\title{
Ischemic Preconditioning Attenuates Morphological and Biochemical Changes in Hepatic Ischemia/Reperfusion in Rats
}

\author{
Li-Ming Jin Yuan-Xing Liu Lin Zhou Hai-Yang Xie Xiao-Wen Feng Hui Li \\ Shu-Sen Zheng
}

Key Laboratory of Combined Multiorgan Transplantation, Ministry of Public Health; Division of Hepatobiliary and Pancreatic Surgery; First Affiliated Hospital, Zhejiang University School of Medicine, Hangzhou, China

\section{Key Words}

Ischemia reperfusion injury $\cdot$ Ischemic preconditioning •

Apoptosis $\cdot$ Leukocyte $\cdot$ Liver

\begin{abstract}
Objective: Ischemic preconditioning (IPC) has been gradually introduced into clinical liver surgery and transplantation in recent years. However, the protective effects of IPC on hepatic warm ischemia/reperfusion (I/R) injury and the potential mechanisms involved are not fully understood. We aimed to evaluate whether the reduction of apoptotic sinusoidal endothelial cells (SECs), induced by IPC, contributes to its protective effect. Methods: Male Wistar rats were randomized into three experimental groups: the continuous clamping group underwent $60 \mathrm{~min}$ of $70 \%$ hepatic ischemia; the IPC group received $10 \mathrm{~min}$ ischemia followed by $10 \mathrm{~min}$ reperfusion prior to ischemia, and the sham control (sham) underwent a sham operation without ischemia. Hepatocyte and SEC apoptosis, liver necrotic areas and the levels of alanine aminotransferase (ALT), aspartate aminotransferase (AST), hyaluronic acid, tumor necrosis factor, myeloperoxidase (MPO) and malondialdehyde were determined. Expression of cysteine-aspartic acid protease-3 (caspase-3) in hepatocytes and SECs was also investigated. Furthermore, the hepatic leukocyte infiltration was assessed by intravital fluorescence microscopy. Results: IPC exhibited a significant alleviation of their postischemic liver function. Serum AST, ALT
\end{abstract}

and tissue MPO were significantly decreased by IPC, and the degree of hepatocyte and SEC apoptosis was significantly inhibited, as shown by the decreased numbers of adherent leukocytes. Conclusions: IPC attenuates hepatic I/R injury by the reduction of leukocyte infiltration, the reduction hepatic enzymatic leakage and the depression of apoptotic cells. SECs are more sensitive to apoptosis induced by warm I/R injury compared to hepatocytes.

Copyright $\odot 2010$ S. Karger AG, Basel

\section{Introduction}

Hepatic ischemia/reperfusion (I/R) injury occurs in trauma, hemorrhagic and cardiac shock, vascular diseases and hepatic surgery, including liver tumor resection and liver transplantation [1-5]. In the transplantation setting, I/R injury plays an essential role in the initial poor function and consequent failure of many liver grafts [6-8]. To date, most investigations have always focused on cold ischemia (cold preservation). However, warm ischemia injury also plays a significant role before and after liver transplantation, especially living liver transplantation. The reduction of warm ischemia may contribute to improving graft function.

As a powerful strategy of experimentally and clinically reducing damage after I/R [3,9-15], ischemic preconditioning (IPC), a well-established phenomenon induced

\section{KARGER}

Fax +4161306 1234

E-Mail karger@karger.ch

www.karger.com
(C) 2010 S. Karger AG, Basel

$1015-2008 / 10 / 0773-0136 \$ 26.00 / 0$

Accessible online at:

www.karger.com/pat
Dr. Shu-Sen Zheng

Key Laboratory of Combined Multiorgan Transplantation

Ministry of Public Health, First Affiliated Hospital

Zhejiang University School of Medicine, Hangzhou 310003 (China)

Tel. +865718723 6567, Fax +86 5718723 6884, E-Mail shusenzheng@zju.edu.cn 
by brief periods of $I / R$, can render an organ resistant to subsequent episodes of more prolonged ischemia. IPC was first described by Murry et al. [16]. They used several brief episodes of myocardial ischemia prior to pretreated ischemia in an animal heart model. Since then IPC has shown great promise for organ-protective strategies and is considered the gold standard [17]. Subsequently, a vast number of investigations have shown the protective effect of IPC on hepatic I/R injury [1, 3, 18-21]. Furthermore, previous studies have focused on sinusoidal endothelial cells (SECs) and their vulnerability in cold I/R injury. Hence SECs are believed to be the primary target cells during this injury $[22,23]$. However, whether or not SECs will also be more susceptible in warm I/R injury compared to other nonparenchymal cells and hepatocytes has not been investigated thoroughly [24], and the potential mechanisms are not completely understood. Furthermore, whether the reduction of apoptotic SECs induced by IPC contributes to its protective effect in warm ischemia has not yet been proven. Based on these hypotheses, we assessed and compared the extent of the injury and the type of cells in the liver that undergo apoptosis and/or necrosis at various time points following warm I/R injury in a rat model randomized or not randomized to IPC. We also touched upon the possible protective mechanism. Our study will help to further understand the mechanisms of warm injury to the graft before liver transplantation, especially in living donor liver transplantation.

\section{Materials and Methods}

\section{Animals}

Male Wistar rats aged between 8 and 10 weeks, weighing between 220 and $260 \mathrm{~g}(\mathrm{n}=75)$, were purchased from Shanghai Animal Center (Chinese Academy of Science, Shanghai, China). The animals were fed a standard laboratory diet with water and food ad libitum. They were kept under constant environmental conditions with a 12-hour light-dark cycle. All animal experiments were approved by the Animal Care Committee of Zhejiang University in accordance with the Principles of Laboratory Animal Care (NIH publication No. 85-23, revised 1985).

\section{Experimental Design}

The animals were randomized into three experimental groups: the continuous clamping group (CC) underwent $60 \mathrm{~min}$ of $70 \%$ hepatic ischemia; the IPC group received $10 \mathrm{~min}$ of ischemia followed by $10 \mathrm{~min}$ reperfusion prior to ischemia, and the sham control underwent a sham operation without ischemia.

\section{Surgical Procedure}

Animals were acclimatized for at least 3 days before surgery. Food was stopped $12 \mathrm{~h}$ before surgery. All surgical procedures were performed under general anesthesia using sodium pentobarbital $60 \mathrm{mg} / \mathrm{kg}$ via an intraperitoneal injection. After a midline laparotomy had been performed, partial hepatic ischemia was induced by clamping the portal vein, hepatic artery and bile duct supplying the median and left lobes of the liver with a microvascular clip, which accounts for $70 \%$ of the rat liver mass [2]. Heparin $100 \mathrm{IU}$ was administered via the caudal vein. As the blood supply to the omental and right lobes remained uninterrupted, intestinal congestion was avoided during clamping by the bypass portal flow through the nonischemic lobes. The duration of the partial hepatic ischemia was selected based on a pilot experiment, in which $60 \mathrm{~min}$ of ischemia caused pronounced liver injury without any fatal accidents, such as severe liver failure or death of the animals. After $60 \mathrm{~min}$ of partial hepatic ischemia, the clip was removed to initiate hepatic reperfusion and the abdominal cavity was closed with a 4-0 silk suture. The animals were allowed to recover from the anesthesia and given free access to food and water. The sham-operated group underwent the same procedures without clamping the pedicle of the liver lobes. The animals were sacrificed after $I / R$ injury at five sampling time points $(0.5,2,6$, $12,24 \mathrm{~h}, \mathrm{n}=5$ /time point). A blood sample, collected from the inferior vena cava of each rat at kill, was centrifuged, and the serum was stored at $-80^{\circ} \mathrm{C}$ until analysis.

\section{IPC Protocol}

IPC included a $10-\mathrm{min}$ period of ischemia followed by $10 \mathrm{~min}$ reperfusion before $60 \mathrm{~min}$ ischemic insult. Briefly, a microvascular clamp was placed on the left lateral lobe for the ischemic period. The clamp was then removed to allow reperfusion for the required period before replacing it for the second ischemic insult. The animal was kept anesthetized for the entire period of preconditioning. After the clamp had been replaced for the second insult, the incision was closed and the animal was allowed to recover.

\section{Biochemical Determinations}

TNF- $\alpha$, Hyaluronic Acid, Aspartate Aminotransferase and

Alanine Aminotransferase Assay

To assess liver damage, hepatic injury was evaluated by determination of the alanine aminotransferase (ALT) and aspartate aminotransferase (AST) levels using an Automated Chemical Analyzer (7600; Hitachi, Tokyo, Japan). Serum hyaluronic acid (HA) levels were measured using the radioimmunoassay method (SHN Medical Research Center, Shanghai, China). Serum TNF- $\alpha$ levels were assayed with enzyme-linked immunosorbent assay (ELISA). The TNF- $\alpha$ ELISA kit was purchased from R\&D Co., USA.

\section{Myeloperoxidase and Malondialdehyde Assay}

For assessment of neutrophil sequestration and activation in the ischemic liver, the myeloperoxidase (MPO) contents were measured. Lipid peroxidation, an indicator of oxidative injury, was determined by measuring the formation of malondialdehyde (MDA). The liver tissue MPO and MDA levels were assayed using commercial kits (NJJC Bio Inc., Nanjing, China) according to the manufacturer's instructions.

\section{Morphometric Assessment of I/R Injury}

Hematoxylin-Eosin Staining

Partial specimens of the left lateral and median lobes were fixed in $10 \%$ formaldehyde and embedded in paraffin. Hematoxylin and eosin (HE)-stained sections $(4 \mu \mathrm{m})$ were evaluated at 
$400 \times$ magnification. Thirty randomly chosen high-power fields were viewed and the presence of hepatic necrotic areas per highpower field was calculated using an Image-Pro Plus software, version 6.0 (Media Cybernetics Inc., Bethesda, Md., USA).

Immunohistochemical Staining of Caspase-3

Tissue sections were deparaffinized by three xylene washes for $5 \mathrm{~min}$. The specimens were hydrated by washing the slides for 5 min with the following solutions: absolute ethanol, twice; $95 \%$ ethanol, once, and 70\% ethanol and phosphate-buffered saline solution (PBS), once. Quench endogenous peroxidase was blocked by washing with $3 \%$ hydrogen peroxide diluted in PBS for $5 \mathrm{~min}$ at room temperature. Epitopes were recovered by heat. Tissue sections were incubated overnight at $4^{\circ} \mathrm{C}$ with antiprotein caspase- 3 primary antibody (bs-0081R, BJBS Biotechnology Co., Beijing, China) diluted 1:150. Tissue sections were then washed in PBS and incubated with secondary anti-rabbit antibody in a 1:200 dilution (BJZS Corp., Beijing, China). They were then incubated for $45 \mathrm{~min}$ in avidin and biotin bound to peroxidase diluted in $5 \mathrm{ml}$ of buffered solution. To identify the reaction, tissue sections were treated with diaminobenzidine solution at a concentration of $1 \mathrm{mg} / \mathrm{ml}$ of buffered solution and hydrogen peroxide for $5 \mathrm{~min}$.

Specimens were counterstained in methyl green for $5 \mathrm{~min}$, submitted to dehydration with absolute ethanol 5 times, and washed in xylene 3 times. For the negative control the primary antibody was not added to one slide of the positive control tissue. Thirty randomly chosen high-power fields were viewed and the number of positive hepatocytes and SECs per high-power field was calculated independently.

\section{TUNEL Staining}

Cleavage of genomic DNA during apoptosis yields DNA strand breaks that can be identified by labeling free 38-hydroxyl ends with modified nucleotides in an enzymatic reaction involving terminal deoxynucleotidyl transferase. Paraffin-embedded sections were prepared and stained for apoptotic cells by the terminal transferase-mediated dUTP nick end-labeling (TUNEL) method using a commercially available kit (Apop Tag Peroxidase In Situ Apoptosis Detection Kit S7100; Chemicon International Inc., Billerica, Mass., USA). The TUNEL assay was performed according to the manufacturer's instructions. Apoptotic hepatocytes and SECs were examined by light microscopy at a magnification of $400 \times$ (high-power field) in a blinded fashion. Only cells with stained nuclei were considered positive for the assay. Data were expressed as the mean of TUNEL-positive cells found per highpower field. A total of 30 random fields were counted for each TUNEL-stained tissue sample. Both the number of hepatocytes and the number of SECs were counted independently.

\section{Intravital Fluorescence Microscopy}

To further understand neutrophil infiltration in the liver, we applied intravital fluorescence microscopy (IFM) and observed the adherent leukocyte in living liver. Liver leukocyte infiltration was assessed at 2, 12 and $24 \mathrm{~h}$ after reperfusion by IFM using a previously described method $[2,25]$. IFM was performed using a modified Olympus microscope (IX81WI; Olympus Optical Corp. $\mathrm{GmbH}$, Hamburg, Germany). Leukocytes were labeled in vivo with rhodamine 6G (i.v. $0.1 \mathrm{ml}, 4 \mathrm{mg} / \mathrm{ml}$; Sigma Chemical Co., St. Louis, Mo., USA) after contrast enhancement with fluorescein-isothiocyanate-labeled dextran (FITC-dextran; i.v. $0.4 \mathrm{ml}, 4 \mathrm{mg} / \mathrm{ml}$;
Sigma Chemical Co.). Ten postsinusoidal venules with connecting sinusoids were evaluated in each animal ( $n=5$ /group) [2].

\section{Statistical Analysis}

All values were expressed as mean $\pm \mathrm{SD}$. Data were analyzed using the SPSS 16.0 software package. Statistical significance between two groups of parametric data was evaluated by one-way ANOVA, and a Student-Newman-Keuls posttest was used for estimation of the stochastic probability in intergroup comparisons. Differences at $\mathrm{p}<0.05$ were considered statistically significant.

\section{Results}

Biochemical Assessment

Assessment of Serum ALT Levels

A significant ALT increase was observed in the CC group at $0.5 \mathrm{~h}$ after reperfusion, whereas a minor increase was seen in the IPC group. The maximal effect was observed at 2 and $6 \mathrm{~h}$ after reperfusion with a 2 -fold reduction in the IPC group. The serum ALT levels of IPC and CC then gradually decreased to normal levels after 6$24 \mathrm{~h}$ reperfusion (fig. 1a).

\section{Assessment of Serum AST Levels}

As shown in figure $1 b$, significant increases in the serum AST activity was observed after I/R from 0.5 to $12 \mathrm{~h}$ in the CC group compared with the sham group, indicating impaired liver function after I/R injury. In contrast, the serum AST activity at 2 and $6 \mathrm{~h}$ was significantly lower in the IPC group (fig. 1b).

\section{Assessment of Serum HA Levels}

In the CC group, a significant increase was observed after 2-12 h of reperfusion compared to the sham group, indicating impaired SEC function after I/R injury. No significant differences were evaluated between the two groups (fig. 1c). The HA changes of the CC group and the IPC group were statistically nonsignificant followed by stably increasing levels for the rest of the study period. In the CC group, a major increase was observed after reperfusion from 0.5 to $24 \mathrm{~h}$. HA levels in IPC tended to be lower than those in the CC group.

\section{Assessment of Serum TNF- $\alpha$ Levels}

The TNF- $\alpha$ level remarkably increased immediately after reperfusion and remained at high levels in the $\mathrm{CC}$ group compared to the IPC group, indicating that IPC can decrease serum TNF- $\alpha$ levels. In contrast, an exchange of the TNF- $\alpha$ level in the CC and the IPC groups after $6 \mathrm{~h}$ reperfusion was identified, and at $24 \mathrm{~h}$, the difference reached statistical significance (fig. 1d). 


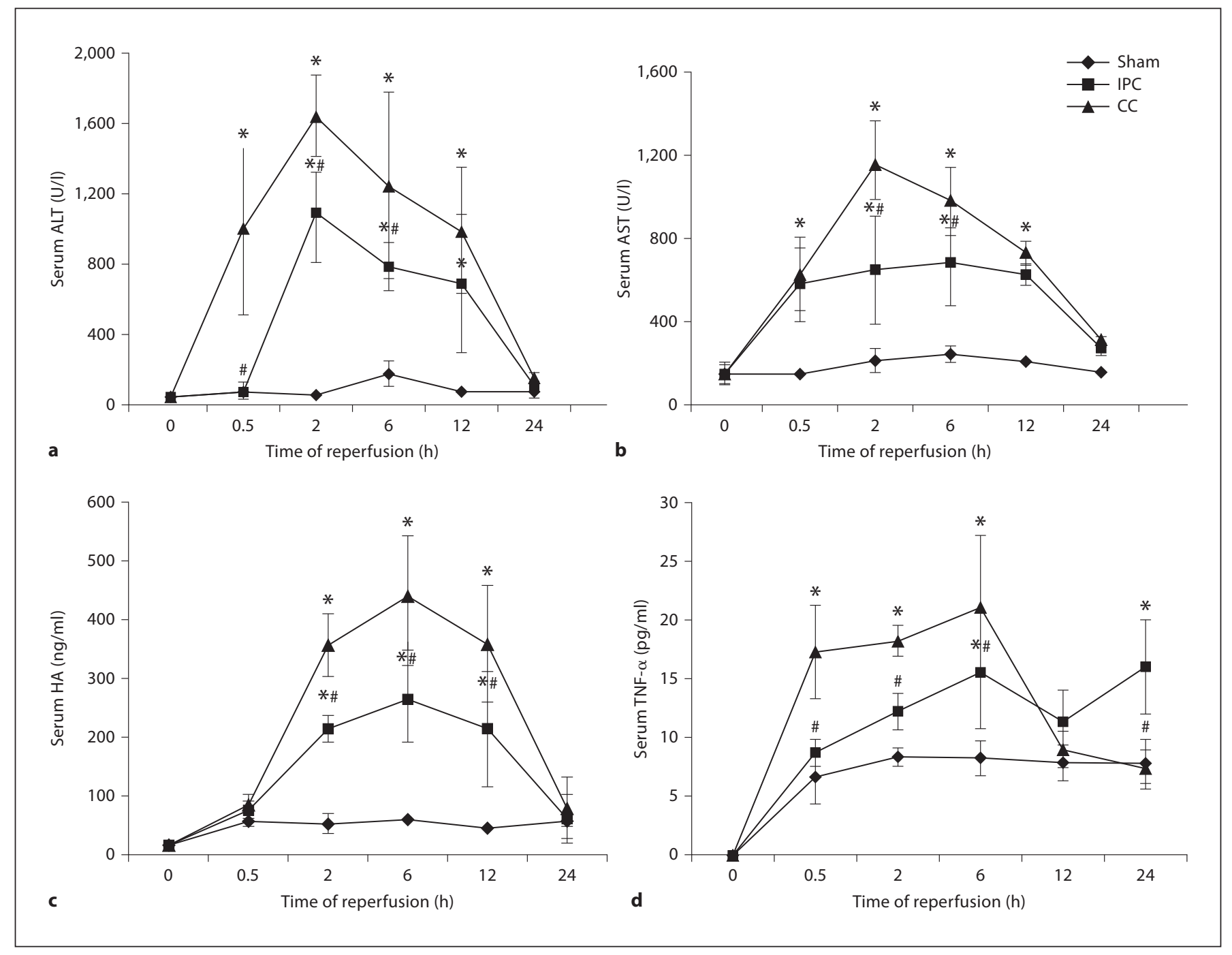

Fig. 1. Changes of serum biochemical marker after $0.5-24 \mathrm{~h}$ of reperfusion. The peak of ALT and AST levels can be seen at $2 \mathrm{~h}$ after reperfusion. Compared with the sham group, the rest of the groups showed significant increases in serum AST and ALT levels $(\mathrm{p}<0.05)$, which gradually return to sham levels after $24 \mathrm{~h}(\mathbf{a}, \mathbf{b})$. HA levels in the IPC group tended to be lower than those in the CC group, and the appearance of the serum peak occurred at $6 \mathrm{~h}$. At 2-12 h after reperfusion, serum HA levels were significantly

\section{Assessment of Liver MPO and MDA Levels}

After $0.5-24 \mathrm{~h}$ of reperfusion, the livers were harvested and the tissue levels of MPO and MDA were assessed. Hepatic I/R injury led to an elevation of the tissue MPO levels at 2-24 $\mathrm{h}$ after reperfusion, and the increases in the MPO levels were significantly inhibited by IPC $(\mathrm{p}<0.05)$. The MDA was significantly decreased at $0.5-24 \mathrm{~h}$ after reperfusion in the IPC group compared to the CC group $(\mathrm{p}<0.05)$ (tables 1, 2). decreased in the IPC group compared to the CC group (c). TNF- $\alpha$ levels significantly increase immediately from 0.5 to $6 \mathrm{~h}$ after reperfusion in the CC group compared to the IPC group. In contrast, TNF- $\alpha$ levels rose in the IPC and decreased in the CC group at $24 \mathrm{~h}$ after reperfusion. At $24 \mathrm{~h}$, the difference of changes from these groups reached statistical significance $(\mathrm{p}<0.05)(\mathbf{d})$. Values are presented as mean $\pm S D$. ${ }^{*} \mathrm{p}<0.05$ vs. sham group; ${ }^{\#} \mathrm{p}<0.05$ vs. CC group.

\section{Histological Evaluation}

\section{HE Staining}

No pathologic changes were observed in liver samples at $6 \mathrm{~h}$ after the sham operation. Only few nuclear condensations and fragmentations of the type associated with apoptotic cell death were focally identified in SECs and hepatocytes. Liver necrotic areas can be calculated on HE-stained tissue section. Other evidence of liver injury was absent or minimal at $6 \mathrm{~h}$ after perfusion (fig. 2). 

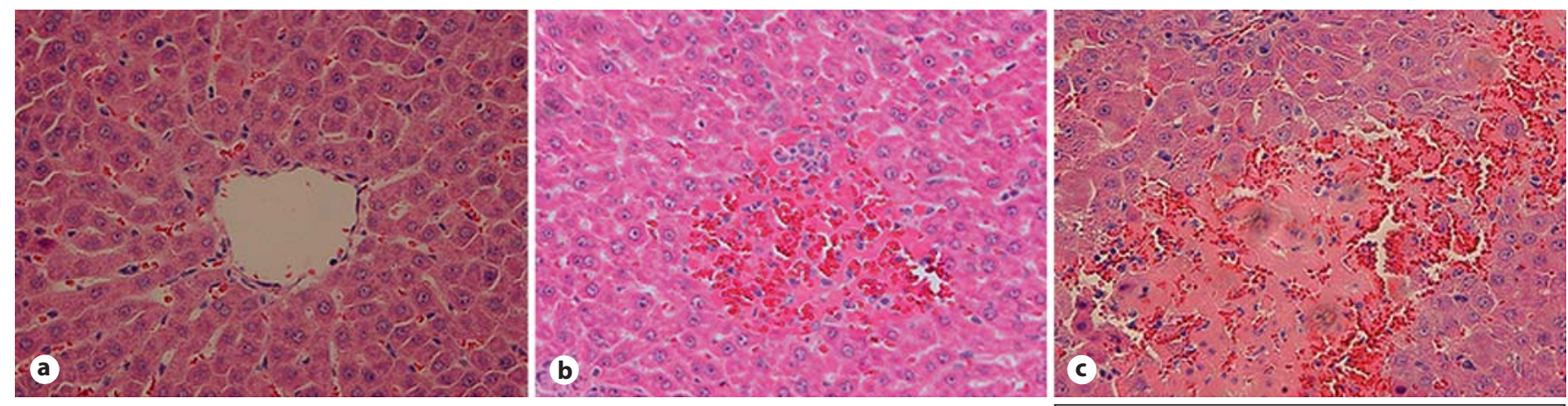

Fig. 2. Presence of hepatic necrotic areas on $\mathrm{HE}$ at $6 \mathrm{~h}$ after reperfusion. There was a significant difference in the presence of necrotic areas between the IPC (b) and the CC group (c). a Shows normal liver construction. ${ }^{*} \mathrm{p}<0.05$ vs. sham group (d).

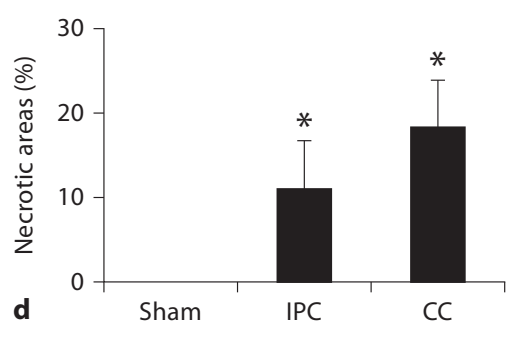

Table 1. Tissue MPO contents at various reperfusion times (U/g wet tissue)

\begin{tabular}{llllll}
\hline & $0.5 \mathrm{~h}$ & $2 \mathrm{~h}$ & $6 \mathrm{~h}$ & $12 \mathrm{~h}$ & $24 \mathrm{~h}$ \\
\hline Sham & $0.62 \pm 0.04$ & $0.61 \pm 0.05$ & $0.56 \pm 0.03$ & $0.90 \pm 0.30$ & $0.73 \pm 0.13$ \\
IPC & $0.94 \pm 0.05^{*}$ & $0.65 \pm 0.14$ & $0.87 \pm 0.16$ & $1.02 \pm 0.04$ & $1.45 \pm 0.25$ \\
CC & $0.95 \pm 0.06$ & $1.05 \pm 0.15^{*, \#}$ & $1.64 \pm 0.32^{*, \#}$ & $1.95 \pm 0.08^{*, \#}$ & $2.11 \pm 0.45^{\#}$ \\
\hline
\end{tabular}

Data represent mean $\pm \mathrm{SD}$ of $\mathrm{n}=5$ rats/group. ${ }^{*} \mathrm{p}<0.05$ vs. sham group; ${ }^{*} \mathrm{p}<0.05$ vs. IPC group.

Table 2. Tissue MDA contents at various reperfusion times (nmol/mg protein)

\begin{tabular}{llllll}
\hline & $0.5 \mathrm{~h}$ & $2 \mathrm{~h}$ & $6 \mathrm{~h}$ & $12 \mathrm{~h}$ & $24 \mathrm{~h}$ \\
\hline Sham & $1.96 \pm 0.09$ & $1.99 \pm 0.22$ & $1.94 \pm 0.08$ & $1.98 \pm 0.03$ & $2.07 \pm 0.16$ \\
$\mathrm{IPC}$ & $2.21 \pm 0.10$ & $2.46 \pm 0.10$ & $2.99 \pm 0.24$ & $2.82 \pm 0.06^{*}$ & $2.88 \pm 0.41$ \\
$\mathrm{CC}$ & $3.58 \pm 0.27^{*, \#}$ & $3.89 \pm 0.19^{*, \#}$ & $4.55 \pm 0.09^{*, \#}$ & $3.58 \pm 0.22^{*}$ & $3.96 \pm 1.55^{*, \#}$ \\
\hline \multicolumn{4}{r}{ Data represent mean \pm SD of $\mathrm{n}=5$ rats/group. ${ }^{*} \mathrm{p}<0.05$ vs. sham group; ${ }^{*} \mathrm{p}<0.05$ vs. IPC group. } \\
\hline
\end{tabular}

\section{TUNEL Staining}

As shown in figure 3, apoptosis appeared in the early phase (0.5-2 h) of reperfusion in both SECs and hepatocytes. Its peak was reached at 6-12 h after reperfusion. Hepatocytes showed changes in apoptosis levels later than SECs. A smaller number of TUNEL-positive SECs were detected in the IPC group, whereas a greater num- ber of positive cells were detected in the CC group. These histological changes were associated with serum ALT and AST levels. However, it was noteworthy that the magnitude of apoptosis in SECs (3.5 positive SECs/high field) was approximately 50 times greater than that observed in hepatocytes (1.5 positive hepatocytes/high field) (fig. 4). 

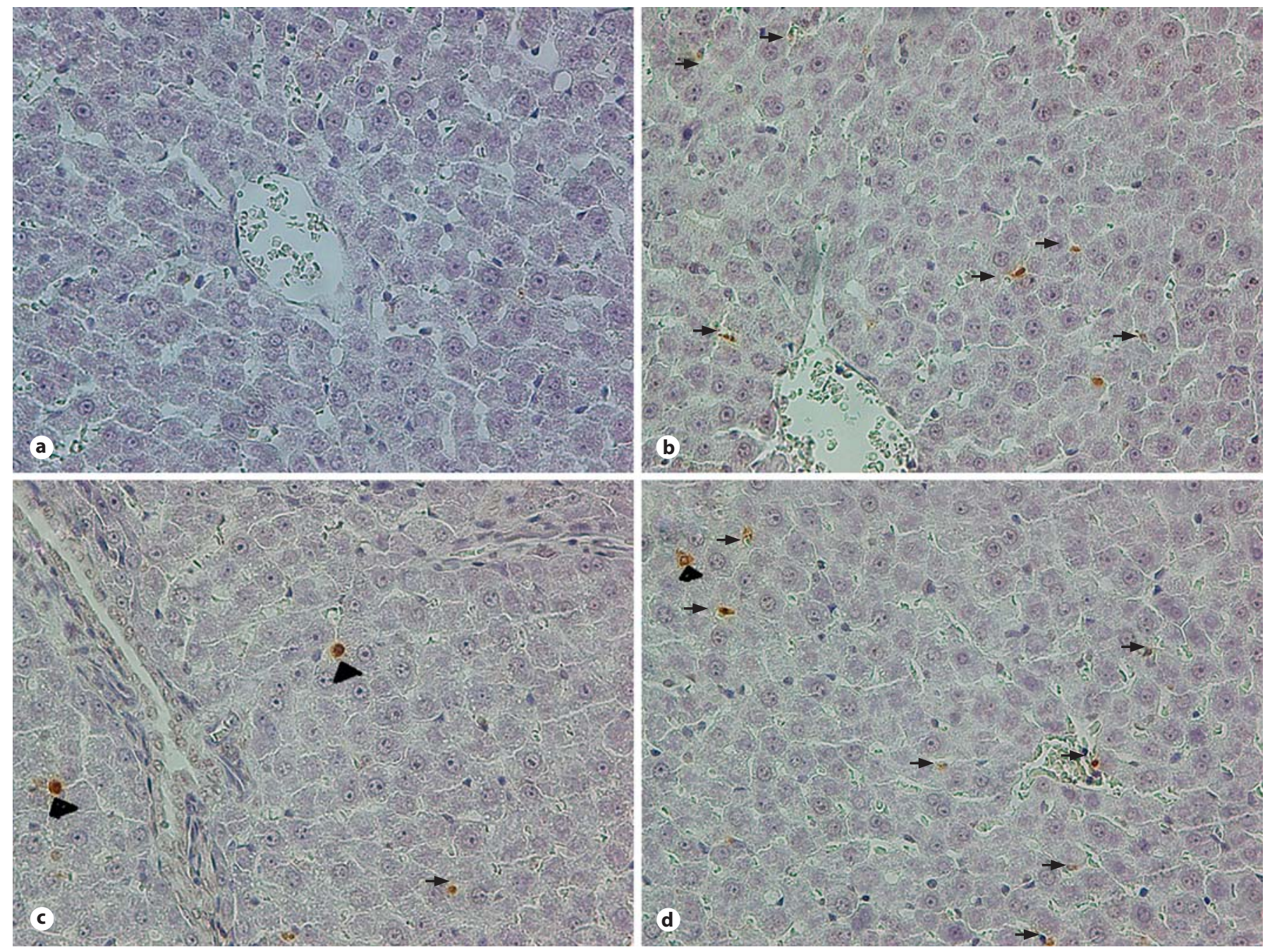

Fig. 3. Detection of apoptotic cells using the TUNEL method at $2 \mathrm{~h}$ after reperfusion. These pictures show massive staining of positive cells in hepatic tissues in 2-hour CC group (b, c, d) and

few positive cells in the sham group (a). Among the positive cells, massive apoptotic SECs (arrows) appeared earlier than apoptotic hepatocytes (arrowheads).

\section{Immunohistochemical Staining of Caspase-3}

In these groups, few positive caspase- 3 cells were observed immediately after reperfusion. After 2-12 h of reperfusion, positive expression of caspase- 3 was observed in massive SECs, but only occasionally in hepatocytes. Peak expression occurred in the 6th hour. Major apoptotic areas were located around central veins. At $24 \mathrm{~h}$, caspase-3 activation on SECs disappeared, and small numbers of hepatocytes were positive for caspase-3 immunostaining (fig. 5).

\section{Assessment of Leukocyte Infiltration in Living Liver}

As shown in figure 6, leukocyte infiltration was blockaded in the IPC group compared with the CC group at

$2-12 \mathrm{~h}$ after reperfusion $(\mathrm{p}<0.05)$, indicating that leukocyte infiltration may participate in the induction of hepatic injury in the early phase (fig. 6).

\section{Discussion}

IPC, introduced by Murry et al. [16] in 1986, has shown great promise in organ-protective strategies $[3,14,26]$. A short period of I/R protects various tissues against subsequent sustained ischemic insults. Previous studies $[4,14$, 27] show the apoptosis of hepatocytes and SECs to be a critical mechanism of injury in the ischemic liver. The apoptosis of SECs has played a pivotal role in cold preser- 

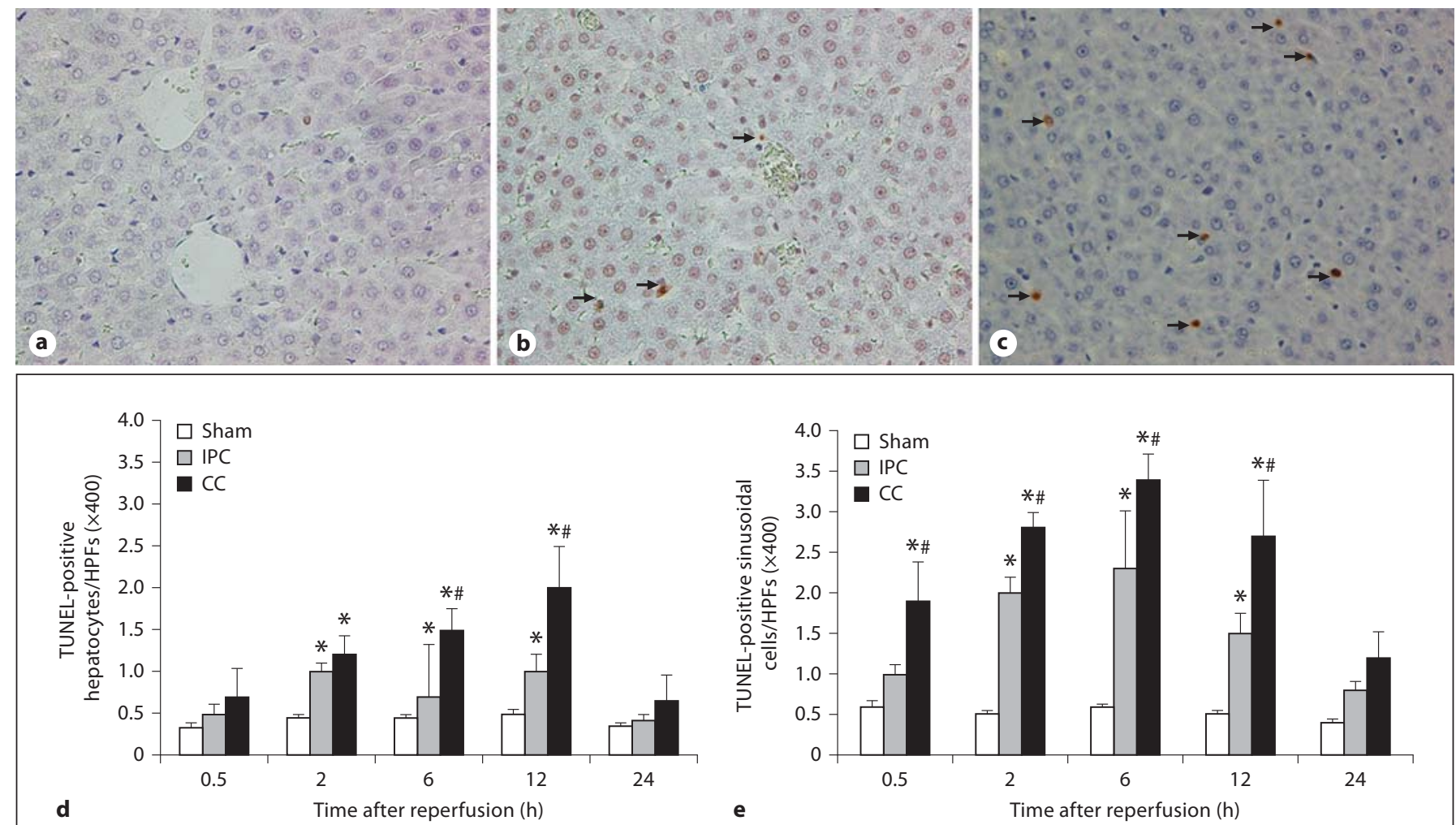

Fig. 4. Evidence of hepatocyte and SEC apoptosis was assessed by the TUNEL assay. In situ TUNEL staining of livers subjected to $60 \mathrm{~min}$ of ischemia and 6-24 h of reperfusion showed no apoptotic cells in the sham group (a) and only rare cells stained positive in the IPC group (b). In contrast, massive staining of positive cells is visible in the CC group (c). Among the positive cells, massive SECs and few hepatocytes appeared at different time points $(\mathbf{d}, \mathbf{e})$. Values are mean $\pm \mathrm{SD} .{ }^{*} \mathrm{p}<0.05$ vs. sham group; ${ }^{*} \mathrm{p}<0.05$ vs. IPC group.

vation injury in liver transplantation [28]. In this study, we presumed that SECs may be the initial cells in warm I/R injury as has been described in cold ischemia [22, 23, $29,30]$. To our knowledge, few investigations have focused on SECs after warm ischemia [24, 31].

In liver surgery, most surgeons clamp hepatic blood supply segmentally rather than totally $[3,32]$. In the present study, as an objective analysis of IPC in normothermic ischemia, we applied the partial hepatic ischemia model, allowing us to evaluate a series of changes of related parameters.

HA is a biochemical marker of liver cell function and represents the function of SECs [33]. An increase in serum HA means damage to SECs. To further understand the degree of SECs injury, we not only evaluated histological changes, but assayed serum HA concentrations. In the present study, the difference of HA levels after reperfusion was significant in the CC and the IPC groups, indicating that IPC effectively ameliorated the impairment of SEC function in the early phase after reperfusion. On the other hand, the serum HA levels were consistent with ALT, AST leakage and histological changes of SECs. In the present experiment, IPC significantly decreased the serum ALT and AST concentrations at 2 and $6 \mathrm{~h}$ after I/R, indicating that it effectively ameliorated the impairment of hepatic function in the early stage after reperfusion. When the number of SEC apoptosis reached its peak after reperfusion at $6 \mathrm{~h}$, at the same time, the serum HA levels also reached the peak. Similarly, when the amount of apoptosis of SECs was close to the baseline, HA levels continued to decrease. In the present study, we combined caspase-3 staining with TUNEL assay in order to objectively assess the occurrence of apoptotic cell death in our liver specimens. The conventional DNA fragmentation detection by TUNEL may fail as a specific marker of apoptosis [24]. Caspase- 3 is an established member of the caspase family known to be involved in the final execution phase of apoptosis. In the present study, experimental data showed that 

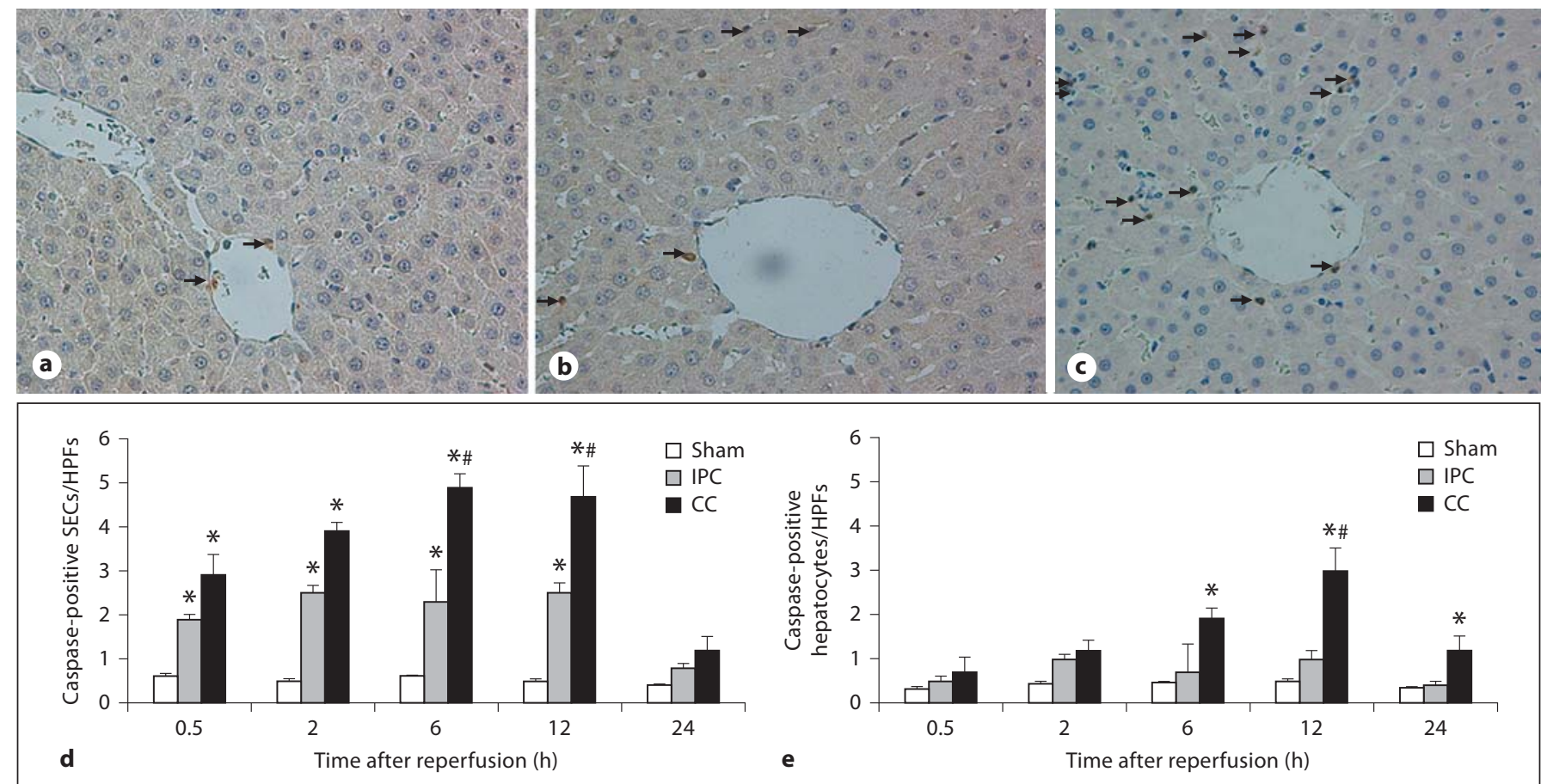

Fig. 5. Morphometric assessment of caspase-3-positive cells at $6 \mathrm{~h}$ after reperfusion. In the sham group, no positive cells were observed (a), only a few cells stained positive in the IPC group (b), and there was massive positive staining of cells in the CC group $(\mathbf{c})(\mathrm{p}<0.05, \mathrm{n}=5$ in each group). Just as TUNEL staining, as the caspase-3 staining indicates massive positive SECs and few hepatocytes at various time points (d, e).

IPC reduced hepatic enzymatic leakage, decreased TNF- $\alpha$ levels after early reperfusion and decreased the number of apopotic SECs compared with the CC group. The reduction of SEC apoptosis was remarkable, and the appearance of apoptotic SECs occurred earlier than that of hepatocyte apoptosis. However, Tejima et al. [34] found that IPC decreased injury in hepatocytes via direct protection of hepatocytes, which is mediated by the suppression of Kupffer cell activation, not via suppression of changes in SECs. We do not explain the exact reasons for this disparity but provide the following possibilities. These conflicting findings may vary depending on the length and type of ischemia, the scope of the ischemic area, and whether or not the organ was excised. Cell death is the key form of hepatic ischemia injury; the ultimate fate of the postischemic liver relies on the balance between cell necrosis or apoptosis and hepatocyte proliferation. Previous observations show that $50-70 \%$ of endothelial cells and $40-60 \%$ of hepatocytes appear to undergo apoptosis during I/R rather than cell necrosis $[24,28,35]$. In the present study, the occurrence of apoptotic cells is associated with inflammatory reactions and reactive oxygen species (ROS) stress, which result in the activation of the apoptotic pathway mediated by mitochondria. Most recently, Arab et al. [36] demonstrated that hepatic parenchyma is able to resist against some types of I/R injury and parenchymal cells are not susceptible to I/R injury, indicating the cell death by apoptosis is the most important change in the early phase after hepatic reperfusion injury.

In the present study, a number of apoptotic cells did not increase remarkably after reperfusion in the CC group, partly because $60 \mathrm{~min}$ of $70 \%$ hepatic warm ischemia is insufficient to induce heavy liver injury. Additionally, we also observed that most of the distribution of apoptosis cells and necrotic areas were located around zone 3 of the liver lobule, indicating that the cells of this site are more susceptible to reperfusion. Although IPC significantly decreased cell apoptosis, IPC failed to abolish the occurrence of cell necrosis after reperfusion. In our study, we observed that liver necrosis was partially inhibited by IPC, although the differences of the necrotic areas failed to reach statistical significance when compared to the CC group. Furthermore, IPC significantly reduced tissue MPO concentrations, whereas CC caused a remarkable increase in MPO levels after reperfusion from 2 to $24 \mathrm{~h}(\mathrm{p}<0.05)$. MPO is a hemoprotein that is 


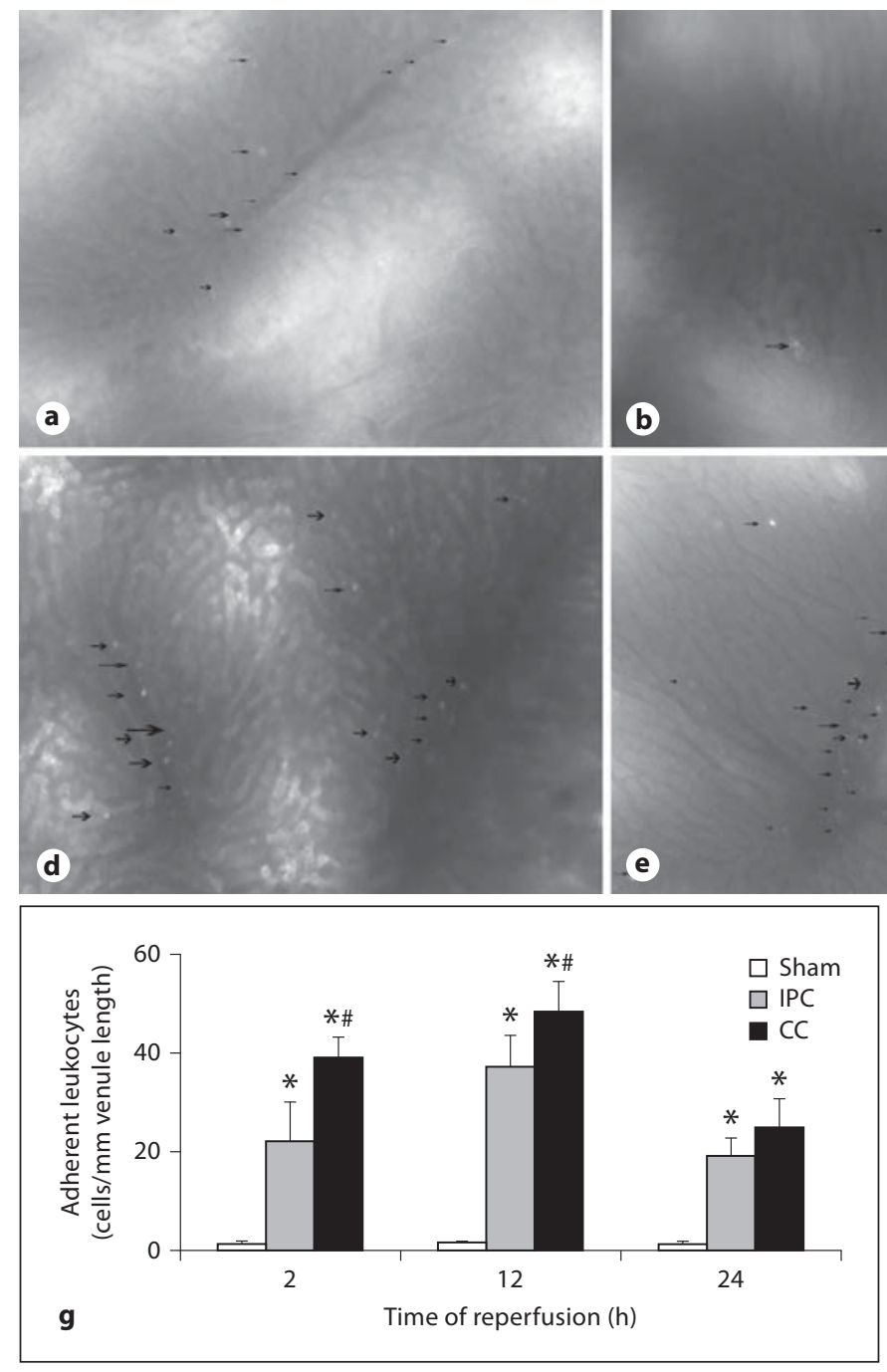

Fig. 6. Alterations of hepatic microcirculation in the CC and IPC groups. Adherent leukocytes and sinusoidal sequestration of leukocytes at $2 \mathrm{~h}$ after reperfusion in the IPC (a) and CC groups (d). Adherent leukocytes and sinusoidal sequestration of leukocytes at $12 \mathrm{~h}$ after reperfusion in the IPC (b) and CC groups (e). Adherent leukocytes at $24 \mathrm{~h}$ after reperfusion in the IPC (c) and CC groups (f). The numbers of adherent leukocytes in postsinusoidal venules were $1.4 \pm 0.55,1.6 \pm 0.55$ and $1.6 \pm 0.55$ cells $/ \mathrm{mm}$ ven-

abundantly expressed in neutrophils and secreted during their activation. It possesses potent proinflammatory properties and contributes directly to tissue injury. Liver MPO activity represents a degree of leukocyte infiltration [2]. Beauchamp et al. [37] suggest that IPC protects SECs partly through a decreased adhesion of leukocytes, secondary to a decreased production of endothelial adhesion molecules. Several studies have shown that adhesion molecules are essential for leukocyte attachment and ule length at 2,12 and $24 \mathrm{~h}$ after reperfusion in the sham group. In contrast, the numbers of adherent leukocytes in postsinusoidal venules (22.4 \pm 7.77 and $37 \pm 6.55$ cells $/ \mathrm{mm}$ venule length, 39.0 \pm 4.3 and $48.4 \pm 6.19$ cells $/ \mathrm{mm}$ venule length, respectively) were more pronounced at 2 and $12 \mathrm{~h}$ after reperfusion in the IPC group than in the CC group (g). The data of $24 \mathrm{~h}$ in the CC and IPC groups did not reach significance. Values are mean $\pm \mathrm{SD} .{ }^{*} \mathrm{p}<$ 0.05 vs. sham group; ${ }^{*} \mathrm{p}<0.05$ vs. IPC group.

penetration through SECs and postsinusoidal venules of the postischemic liver [38, 39]. Yuan et al. [40] observed that IPC exerted protective effects on both SECs and hepatocytes during liver I/R injury and explained a mechanism involving a reduction in leukocyte infiltration and modulation of the imbalance of the endogenous oxidantantioxidant system. For further observation of adherent leukocytes in the liver, we applied the IFM technique [2]. In the present study, we found that the inhibitory effects 
of IPC on hepatic function correlated very well with the reduction of adherent leukocytes in the liver. Analysis of leukocyte attachment by IFM revealed that the postischemic increases in the numbers of adherent leukocytes were significantly reduced in the IPC animals. Only a few sequestrated leukocytes were observed in the postsinusoidal venules of sham animals (fig. 6). The number of adherent leukocytes in the CC group was consistent with tissue MPO levels, indicating that leukocyte infiltration plays a significant role during hepatic I/R injury. Furthermore, these observations indicate that IPC has beneficial hemodynamic effects on hepatic I/R by alleviating postischemic leukocyte trapping. As a result, the suppression of leukocyte attachment plays an important role in improving hepatic I/R injury, where IPC has significantly decreased the number of adherent leukocytes.

Usually, oxidative stress is considered a mediator of programmed cell death. SECs are damaged by oxygenfree radicals produced on reperfusion following liver ischemia [41]. Actually, ROS and toxic free radicals have been implicated in the mediation of hepatic I/R injury [12]. In the present study, IPC significantly reduced tissue MDA and MPO levels, indicating that suppression of neutrophil sequestration and activation play a fundamental role in I/R injury by directly or indirectly inducing ROS production. Early hepatic injury after reperfusion is associated with the relevant triggers for apoptotic cell death. TNF- $\alpha$ has been shown to induce the necrosis and apoptosis of liver parenchymal and nonparenchymal cells [42] via stimulating hepatic infiltration of leukocytes [25]. Conversely, IPC can prevent systemic events caused by the release of TNF- $\alpha$ after liver reperfusion
[42-44]. In the present study, we observed that there was a relatively lower, stable level of TNF- $\alpha$ in the IPC group in the early and subacute phases compared with the CC group, and other biological indexes and the degree of histological damage of the IPC group were mild. As a result, the pleiotropic effects of TNF- $\alpha$ may be the relation to the low or high of the serum concentration. Teoh et al. [45] mimicked IPC response by different-dose TNF- $\alpha$ ( 1 and $5 \mu \mathrm{g} / \mathrm{kg}$ body weight) injection to evaluate the injurious and proliferative index, showing that low-dose TNF- $\alpha$ is just as effective at reducing hepatic I/R injury as IPC. In contrast, they observed that higher doses of TNF- $\alpha(25 \mu \mathrm{g} / \mathrm{kg}$ body weight) augment hepatoxicity rather than protect against I/R injury. Therefore, many investigators think that TNF- $\alpha$ has a dual role (apoptosis and proliferation) in both the early and late phases in case of hepatic I/R injury or major tissue loss [45-48].

In summary, the findings in the present study demonstrated that SECs were more sensitive to apoptosis induced by warm $\mathrm{I} / \mathrm{R}$ injury than hepatocytes. IPC conferred protection to hepatic I/R through reducing hepatic enzymatic leakage, reducing cell apoptosis and improving neutrophil infiltration.

\section{Acknowledgments}

This study was supported by the National Basic Research Program of China (973 Program; No. 2009CB522403), the National Key R\&D Program (No. 2008BAI60B02) and the National Science and Technology Major Projects (No. 2008ZX10002-026). We would like to thank Jennifer Meintjes from the University of Western Australia for revising the language.

\section{References}

$>1$ Clavien PA, Selzner M, Rudiger HA, et al: A prospective randomized study in 100 consecutive patients undergoing major liver resection with versus without ischemic preconditioning. Ann Surg 2003;238:843-852.

$>2$ Liu YX, Jin LM, Zhou L, et al: Mycophenolate mofetil attenuates liver ischemia/reperfusion injury in rats. Transpl Int 2009;22:747756.

$\checkmark 3$ Clavien PA, Petrowsky H, DeOliveira ML, et al: Strategies for safer liver surgery and partial liver transplantation. N Engl J Med 2007; 356:1545-1559.

$\checkmark 4$ Yadav SS, Sindram D, Perry DK, et al: Ischemic preconditioning protects the mouse liver by inhibition of apoptosis through a caspase-dependent pathway. Hepatology 1999;30:1223-1231.
5 Breitenstein S, Apestegui C, Petrowsky H, et al: 'State of the art' in liver resection and living donor liver transplantation: a worldwide survey of 100 liver centers. World J Surg 2009;33:797-803.

$\checkmark 6$ Banga NR, Homer-Vanniasinkam S, Graham $\mathrm{A}$, et al: Ischaemic preconditioning in transplantation and major resection of the liver. Br J Surg 2005;92:528-538.

7 DeOliveira ML, Graf R, Clavien PA: Ischemic preconditioning: promises from the laboratory to patients - sustained or disillusioned? Am J Transplant 2008;8:489-491.
8 Kataoka M, Shimizu H, Mitsuhashi N, et al: Effect of cold-ischemia time on C-X-C chemokine expression and neutrophil accumulation in the graft liver after orthotopic liver transplantation in rats. Transplantation 2002;73:1730-1735.

$\checkmark$ Montalvo-Jave EE, Pina E, Montalvo-Arenas $\mathrm{C}$, et al: Role of ischemic preconditioning in liver surgery and hepatic transplantation. J Gastrointest Surg 2009;13:2074-2083.

10 Zahler S, Kupatt C, Becker BF: Endothelial preconditioning by transient oxidative stress reduces inflammatory responses of cultured endothelial cells to TNF-alpha. FASEB J 2000;14:555-564. 
-11 Amador A, Grande L, Marti J, et al: Ischemic pre-conditioning in deceased donor liver transplantation: a prospective randomized clinical trial. Am J Transplant 2007;7:21802189.

-12 Ambros JT, Herrero-Fresneda I, Borau OG, et al: Ischemic preconditioning in solid organ transplantation: from experimental to clinics. Transpl Int 2007;20:219-229.

13 Arkadopoulos N, Kostopanagiotou G, Theodoraki $\mathrm{K}$, et al: Ischemic preconditioning confers antiapoptotic protection during major hepatectomies performed under combined inflow and outflow exclusion of the liver. A randomized clinical trial. World J Surg 2009;33:1909-1915.

14 Franchello A, Gilbo N, David E, et al: Ischemic preconditioning (IP) of the liver as a safe and protective technique against ischemia/reperfusion injury (IRI). Am J Transplant 2009;9:1629-1639.

-15 Suzuki S, Inaba K, Konno H: Ischemic preconditioning in hepatic ischemia and reperfusion. Curr Opin Organ Transplant 2008 13:142-147.

-16 Murry CE, Jennings RB, Reimer KA: Preconditioning with ischemia: a delay of lethal cell injury in ischemic myocardium. Circulation 1986;74:1124-1136.

17 Gumina RJ, Gross GJ: If ischemic preconditioning is the gold standard, has a platinum standard of cardioprotection arrived? Comparison with NHE inhibition. J Thromb Thrombolysis 1999;8:39-44.

-18 Lloris-Carsi JM, Cejalvo D, Toledo-Pereyra LH, et al: Preconditioning: effect upon lesion modulation in warm liver ischemia. Transplant Proc 1993;25:3303-3304.

$\checkmark 19$ Matsumoto T, O’Malley K, Efron PA, et al: Interleukin- 6 and STAT3 protect the liver from hepatic ischemia and reperfusion injury during ischemic preconditioning. Surgery 2006;140:793-802.

20 Ofluoglu E, Kerem M, Pasaoglu H, et al: Delayed energy protection of ischemic preconditioning on hepatic ischemia/reperfusion injury in rats. Eur Surg Res 2006;38:114-121.

$\checkmark 21$ Clavien PA, Yadav S, Sindram D, et al: Protective effects of ischemic preconditioning for liver resection performed under inflow occlusion in humans. Ann Surg 2000;232: 155-162.

22 Caldwell-Kenkel JC, Currin RT, Tanaka Y, et al: Reperfusion injury to endothelial cells following cold ischemic storage of rat livers. Hepatology 1989;10:292-299.

23 Miyagawa Y, Imamura H, Soeda J, et al: Fate of hepatocyte and sinusoidal lining cell function and kinetics after extended cold preservation and transplantation of the rat liver. Liver Transpl 2002;8:370-381.
Kohli V, Selzner M, Madden JF, et al: Endothelial cell and hepatocyte deaths occur by apoptosis after ischemia-reperfusion injury in the rat liver. Transplantation 1999;67: 1099-1105.

25 Klintman D, Li X, Santen S, et al: p38 mitogen-activated protein kinase-dependent chemokine production, leukocyte recruitment, and hepatocellular apoptosis in endotoxemic liver injury. Ann Surg 2005;242: 830-839.

26 Vinten-Johansen J, Zhao ZQ, Jiang R, et al: Preconditioning and postconditioning: innate cardioprotection from ischemia-reperfusion injury. J Appl Physiol 2007;103:14411448

27 Teoh NC, Farrell GC: Hepatic ischemia reperfusion injury: pathogenic mechanisms and basis for hepatoprotection. J Gastroenterol Hepatol 2003;18:891-902.

28 Gao W, Bentley RC, Madden JF, et al: Apoptosis of sinusoidal endothelial cells is a critical mechanism of preservation injury in rat liver transplantation. Hepatology 1998;27: 1652-1660.

29 Arai M, Thurman RG, Lemasters JJ: Ischemic preconditioning of rat livers against cold storage-reperfusion injury: role of nonparenchymal cells and the phenomenon of heterologous preconditioning. Liver Transpl 2001;7:292-299.

30 Arai M, Peng XX, Currin RT, et al: Protection of sinusoidal endothelial cells against storage/reperfusion injury by prostaglandin $\mathrm{E}_{2}$ derived from Kupffer cells. Transplantation 1999;68:440-445.

31 Quireze C, Montero EF, Leitao RM, et al: Ischemic preconditioning prevents apoptotic cell death and necrosis in early and intermediate phases of liver ischemia-reperfusion injury in rats. J Invest Surg 2006;19:229236.

32 Imamura H, Kokudo N, Sugawara Y, et al: Pringle's maneuver and selective inflow occlusion in living donor liver hepatectomy. Liver Transpl 2004;10:771-778.

-33 Shimoda M, Iwasaki Y, Sawada T, et al: Protective effect of ischemic preconditioning against liver injury after major hepatectomy using the intermittent Pringle maneuver in swine. Pathobiology 2007;74:42-49.

- 34 Tejima K, Arai M, Ikeda H, et al: Ischemic preconditioning protects hepatocytes via reactive oxygen species derived from Kupffer cells in rats. Gastroenterology 2004;127: 1488-1496.

35 Cursio R, Gugenheim J, Ricci JE, et al: A caspase inhibitor fully protects rats against lethal normothermic liver ischemia by inhibition of liver apoptosis. FASEB J 1999;13: 253-261.
36 Arab HA, Sasani F, Rafiee MH, et al: Histological and biochemical alterations in earlystage lobar ischemia-reperfusion in rat liver. World J Gastroenterol 2009;15:19511957.

37 Beauchamp P, Richard V, Tamion F, et al: Protective effects of preconditioning in cultured rat endothelial cells: effects on neutrophil adhesion and expression of ICAM-1 after anoxia and reoxygenation. Circulation 1999;100:541-546.

38 Hafez T, Moussa M, Nesim I, et al: The effect of intraportal prostaglandin $\mathrm{E}_{1}$ on adhesion molecule expression, inflammatory modulator function, and histology in canine hepatic ischemia/reperfusion injury. J Surg Res 2007;138:88-99.

39 Teoh NC, Ito Y, Field J, et al: Diannexin, a novel annexin $\mathrm{V}$ homodimer, provides prolonged protection against hepatic ischemiareperfusion injury in mice. Gastroenterology 2007;133:632-646.

40 Yuan GJ, Ma JC, Gong ZJ, et al: Modulation of liver oxidant-antioxidant system by ischemic preconditioning during ischemia/reperfusion injury in rats. World J Gastroenterol 2005;11:1825-1828.

41 Sindram D, Rudiger HA, Upadhya AG, et al: Ischemic preconditioning protects against cold ischemic injury through an oxidative stress dependent mechanism. J Hepatol 2002;36:78-84.

-42 Kannerup AS, Gronbaek H, Funch-Jensen P, et al: Cytokine changes during warm ischemia and reperfusion of the pig liver with or without preconditioning. Eur Surg Res 2009; 42: 216-222.

43 Li XC, Ma YF, Wang XH: Role of NF-kappaB as effector of IPC in donor livers before liver transplantation in rats. Transplant Proc 2006;38:1584-1587.

44 Fernandez L, Carrasco-Chaumel E, Serafin $\mathrm{A}$, et al: Is ischemic preconditioning a useful strategy in steatotic liver transplantation? Am J Transplant 2004;4:888-899.

45 Teoh N, Leclercq I, Pena AD, et al: Low-dose TNF-alpha protects against hepatic ischemia-reperfusion injury in mice: implications for preconditioning. Hepatology 2003; $37: 118-128$

-46 Teoh N, Field J, Sutton J, et al: Dual role of tumor necrosis factor-alpha in hepatic ischemia-reperfusion injury: studies in tumor necrosis factor-alpha gene knockout mice. Hepatology 2004;39:412-421.

47 Taub R: Liver regeneration: from myth to mechanism. Nat Rev Mol Cell Biol 2004;5: 836-847.

48 Selzner N, Selzner M, Odermatt B, et al: ICAM-1 triggers liver regeneration through leukocyte recruitment and Kupffer cell-dependent release of TNF-alpha/IL-6 in mice. Gastroenterology 2003;124:692-700. 